\title{
Communication Architecture of the IEC 61850-based Micro Grid System
}

\author{
Byong-Kwan Yoo*, Seung-Ho Yang*, Hyo-Sik Yang ${ }^{\dagger}$, Won-Yong Kim**, \\ Yu-Seok Jeong**, Byung-Moon Han** and Kwang-Soo Jang***
}

\begin{abstract}
As the power grids are integrated into one big umbrella called a "smart grid," communication protocol plays a key role in successful operations. The successful deployment of smart grid interoperability is a major hurdle that must be overcome. The micro grid, a small power system that distributes energy resource, is operated in diverse regions. Different vendors use different communication protocols in the operation of the micro grid. Recently, the IEC 61850 has been legislated to solve the interoperability problems in power utility automation. The present paper presents a micro grid system based on the IEC 61850 protocol. It consists of a micro grid monitoring system, a protocol converter that transforms serial data to IEC 61850 data, and distributed energy resource controllers for diverse DER nodes. A developed communication gateway can be deployed for DER controllers with serial links to exchange data with IEC 61850-based devices. The gateway can be extended to IEC 61850-based distribution automation systems, substation automation systems, or SCADA.
\end{abstract}

Keywords: Communication System, Gateway, IEC 61850, Protocol Converter, Micro Grid

\section{Introduction}

An old paradigm for the existing power industry is the one-way power flow system, in which power stations only serve as producers of electricity and customers play the role of electricity consumers. However, the paradigm has changed due to the appearance of prosumers that consume and generate electricity at the same time. In the current power system, the prosumers could only support their own needs but not provide electricity, which they could produce and return to the power utility system. This means that overproduced electricity is thrown away and wasted if the energy storages of the prosumers are full.

To surmount this inefficiency, the micro grid was proposed. The smart grid dictionary defines the micro grid as a small power system, which includes self-contained generation, transmission, distribution, sensors, energy storage, and energy management software with a seamless and synchronized connection to a utility power system; however, the micro grid can operate independently as an island from that system [1]. To make a micro grid

$\dagger \quad$ Corresponding Author: Dept. of Computer Science and Engineering, Sejong University, Korea. (hsyang@sejong.ac.kr)

* Dept. of Computer Science and Engineering, Sejong University, Korea. (pkyours andiah063@gmail.com)

** Dept. of Electrical Engineering, Myongji University, Korea. (acorn999@naver.com, and erichan and jeong@mju.ac.kr)

*** LG CNS, Korea. (ksthink@hotmail.com)

Received: February 16, 2011; Accepted: June 2, 201 interoperable to the smart grid, the existing power industry has to be changed from a one-way to a two-way system, and the integration of power and communication technology has to be carefully studied and tested. Interconnection of distributed energy resources (DER) and distribution automation system is the key to better smart grid systems in the future [2].

DER vendors need communication capability because they interconnect to the smart grid. Up until now, DER vendors have used their own proprietary communication protocols due to the lack of standards [3]. The distributed energy resources logical nodes [4] data model for DER are standardized under the basic communication structure stipulated in the communication networks and systems for power utility automation introduced in the IEC 61850-7420. The IEC 61850 is known as flexible and future-proof due to its capability for decoupling domain-specific application data model from the communication protocol stack.

The IEC 61850 has been adopted as an international communication protocol to monitor, control, and measure power utilities. The IEC $61850-7-420$ has been designated to interact with the micro grid. Given that communication protocol has become critical in the smart grid system, mores studies on the communication technology in micro grid environments have to be conducted. Studies on IEC 61850 related to substation and distribution automation system are actively in progress [5-7]. Due to the lack of research on the implementation of the whole micro grid communication system view, proprietary protocols are still 
used mainly in the industry, presenting a serious hurdle. Research efforts on the micro grid have been conducted by means of computer simulation $[8,9]$. Moreover, the micro grid system has been implemented [7, 9-11]. The scope of implementation, however, has been limited to the micro system. General communication protocols, rather than the IEC 61850 used for micro grid, are presented in $[9,10]$. An IEC 61850-based micro grid system is presented in [12]. Meanwhile, IEC 61850-7-420 object models have been employed to model the diesel generator located within the Salt River Project (SRP) distribution systems. Using the Modbus interface, diesel generators with a rating of 93 $\mathrm{kVA}$ at $75 \mathrm{~kW}$ and 0.8 power factor are connected to the SCADA sentry, which is similar to the gateway described in the present paper. Such a system, however, is limited to point-to-point communication link, and does not implement grid operation. The concept behind virtual power plants (VPPs) consisting of the DER unit has been introduced under project EDISON [13]. EDISON uses data model and services defined in IEC 61850 for interoperability. However, it proposes a conceptual serviceoriented architecture and power balance simulation using computer simulation. The design of the application layer protocol, the implementation, and deployment are left for future work. Real Time Digital Simulator (RTDS) was employed in [14] to simulate the micro grid system which did not deploy real micro grid equipment.

The present paper introduces an IEC 61850-based micro grid system, which interconnects several DER units with the micro grid monitoring system. Various real DER units are deployed, including solar cell arrays, fuel cells, and wind turbines, with dedicated DER controller units for monitoring and control. Each DER unit controller is connected to the dedicated protocol converter, i.e., gateway, which transforms proprietary data to standardized IEC 61850 data models. An embedded Linux-based gateway is also developed, which could be deployed by any DER controller through a serial interface. The gateway receives data from DER controllers using their proprietary protocol through a serial cable, transforms it to fit the IEC 61850 data model, and sends it to the micro grid monitoring system using services defined in IEC 61850. The micro grid monitoring system can request data for each DER unit to the gateways as well. For gateways using standardized services and data model defined in IEC 61850, interoperability to existing IEC 61850 -based power grids is guaranteed, including distribution automation systems (DAS), substation automation systems (SAS), or SCADA (Supervisory Control And Data Acquisition) systems. As described previously, current micro grid systems use their own proprietary communication protocols with their own data models; one DER unit is monitored using IEC 61850. In the present paper, however, four different DER units that communicate with standardized communication protocols and standardized data model (IEC 61850) are connected. DER units are monitored using IEC 61850; the units were also controlled. By using standardized communication protocols and data model, any DER unit which conforms to IEC 61850 could be connected to existing micro grid systems, thus providing expandability.

The current study describes an IEC 61850-based micro grid from the communication perspective. The paper is organized into sections. Section 2 describes the standardization effort in power utility systems; focus is given to the IEC 61850 standardization efforts. Section 3 describes the developed micro grid monitoring system and gateway in detail, and Section 4 concludes the paper.

\section{Standardization Efforts for Interoperability in the Power Industry}

In a power utility system, the standards related to the communication system has been provided for years to ensure the exchange of critical information, e.g., control commands, status information, and measurement data, efficiently. Among the standards are Distributed Network Protocol (DNP), UCA 3.0, Modbus, and PROFIBUS, as well as other proprietary protocols with custom communication links. Using these protocols, many solutions from different vendors have been proposed, as shown in Fig. 1. Due to the lack of interoperability among different vendors, substation automation systems (SAS) operators face difficulties and complexities in the management of SAS.

Some solutions based on the old-fashioned communication protocols have been designed under a limited bandwidth. These communication protocols are focused on flow and error control due to the lack of reliability in the communication link. Furthermore, these solutions have not been developed specifically for the power industry, but for the automation system industry. This means that these protocols do not define the data model for the power industry. These solutions are register-based protocols, and every piece of data is assigned to a point-based data model. Register-based protocols are basically designed to minimize the bandwidth, requiring tremendous efforts for system engineering. Eventually, as the amount of information increases, the existing standard reaches the limit of use. In response, the standardization project, IEC 61850, has been initiated to solve these issues in 1995 [15].Originally, IEC 61850 has focused only on SAS. However, the scope of IEC 61850 has been extended in the second edition to cover the entire power utility system. Since the revision, the IEC 61850 has focused on the entire power system, including various views such as the way of engineering, the functionalities (i.e., protection, control, and monitoring), and hundreds of words used for specifying standardized data. Employing the objectoriented notion, all data can be reused, and hierarchical structures are used to access to the data. IEC 61850 offers the common structure of three representative levels of the 

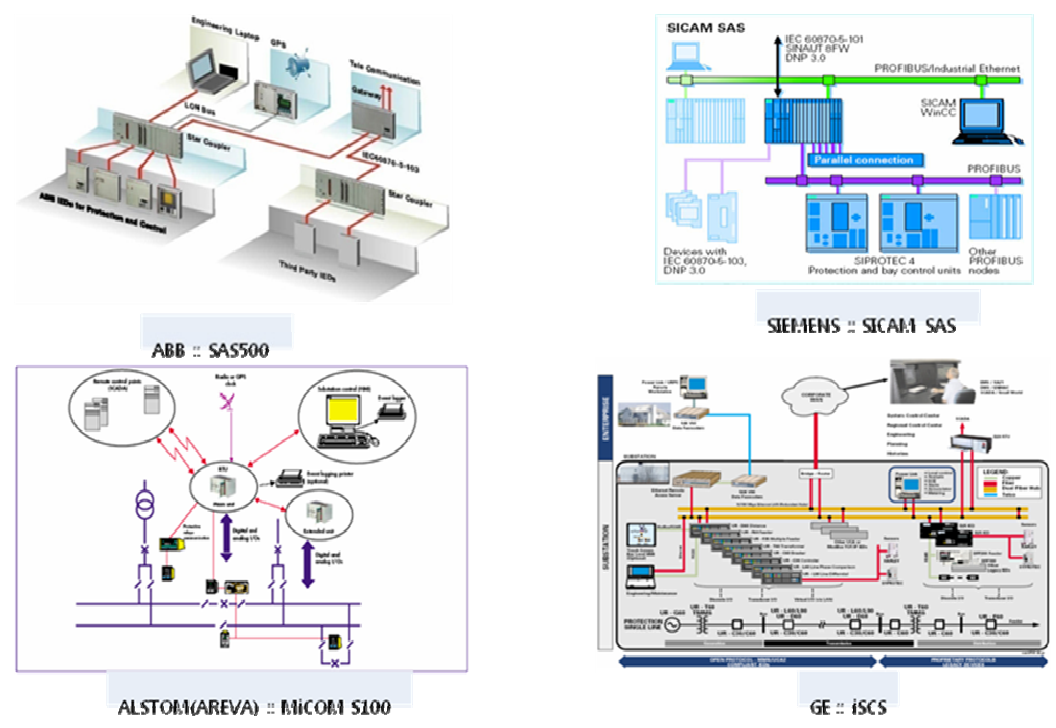

Fig. 1. Vendor dependent solutions

system, namely, the process level (current transformers or CTs/power transformers or PTs and switches), bay level (protection and control devices), and station level (human machine interface (HMI) and remote command link to the SCADA system).

The most significant difference between IEC 61850 and conventional protocols is that the former covers not only existing data models but also a wide scope of data models by providing the basic template for these. In addition, IEC 61850 defines the following:

- general subjects related to the substation, including the project management, environment and the requirement of EMC;

- the principal information related to the main functions and devices, including measurement data, the status, and switching information;

- the information exchange for protection, monitoring, control, measuring and metering;

- the measured digital information exchange for a primary equipment such as a switch, a transformer or a $\mathrm{CT} / \mathrm{PT}$; and

- the substation configuration language (SCL) for system engineering.

An extension of IEC 61850 standard series, the IEC 61850-7-420 on "Communications Standard for DERs," which defines the data model used in micro grids, has been published [4]. The data models for wind power plants are defined separately in [16]. Recently, numerous DER systems are being interconnected to power utility systems for various economic, social, and environmental benefits. Moreover, utilities and DER manufacturers have realized the strong demand to have a single international standard to reduce overall costs, simplify implementation and maintenance, as well as maximize the reliability and efficiency of power systems. Enterprises in DER have used the proprietary protocol and various communication protocols; however, they have established the international standard to solve the problems of interoperability as well as to provide the interface for communication and control.

Fig. 2 shows the notion of Logical Nodes (LN) defined in IEC 61850-7-420. As shown in the figure, most logical nodes defined in IEC 61850-7-4 are reused; in addition, logical nodes specialized for DER are defined in IEC 61850-7-420. Current communication models and engineering processes follow IEC 61850. The definitions of many data models that have not yet been defined but are necessary for the operation of power utility systems, as presented in the first edition of IEC 61850, are currently being developed in the second edition of IEC 61850.

\section{IEC 61850-based Micro Grid System}

Fig. 3 shows the communication architecture of the deployed micro grid system.

The figure shows DER nodes, i.e., wind power generator, photovoltaic system, fuel cells, and energy storage that have dedicated controllers for each DER unit. Each controller sends measured and status data that are encoded with their own proprietary protocol to the dedicated gateway using a serial communication link. The gateways convert received measured values and status information to those prescribed by the IEC 61850 data model and send them to the micro grid monitoring system through a 100 Mbps Ethernet interface using services defined in IEC 61850. The micro grid monitoring system works as a small Energy Management System (EMS), which sends control command to the DER controller via the gateway as required. Each component of the deployed micro system is described in a top-down manner. 


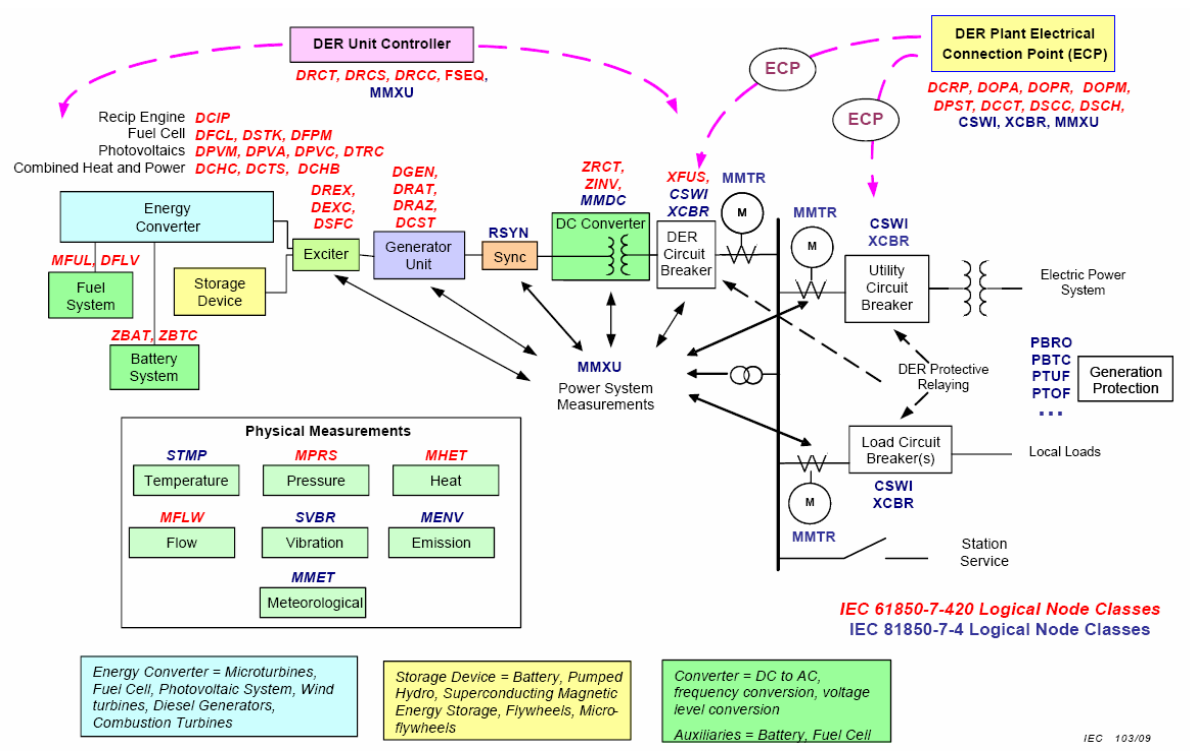

Fig. 2. Conceptual data model of logical nodes in DER in IEC 61850-7-420 [4]

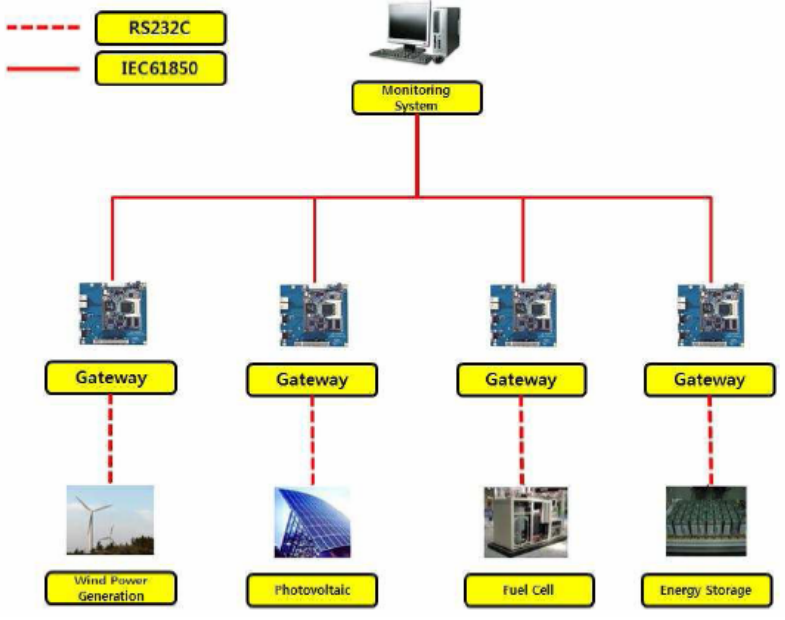

Fig. 3. Micro grid communication architecture

\subsection{Local DER Monitoring System}

DER monitoring systems, which are meant to be in the control room of micro grid systems to monitor the entire micro grid system, have been developed to monitor the DER nodes. Similar IEC 61850 based HMI with SCL viewer was developed in [17]. The HMI, however, cannot connect to multiple servers. These have a very simple graphic user interface (GUI) for intuitive use. The main GUI consists of three sections, three buttons, and multiple taps representing the number of DER units connected to the monitoring system. The three sections are called "Named Variables," "System Information" and "ObjectValue-Type," respectively. Fig. 4 shows the main GUI of the micro grid monitoring system, which is connected to the four DER units, i.e., photovoltaic, wind, fuel cell, and battery charger. The data models and their photovoltaic system values are also shown in Fig. 4. The micro grid monitoring system can connect as many DER units as the system requires.

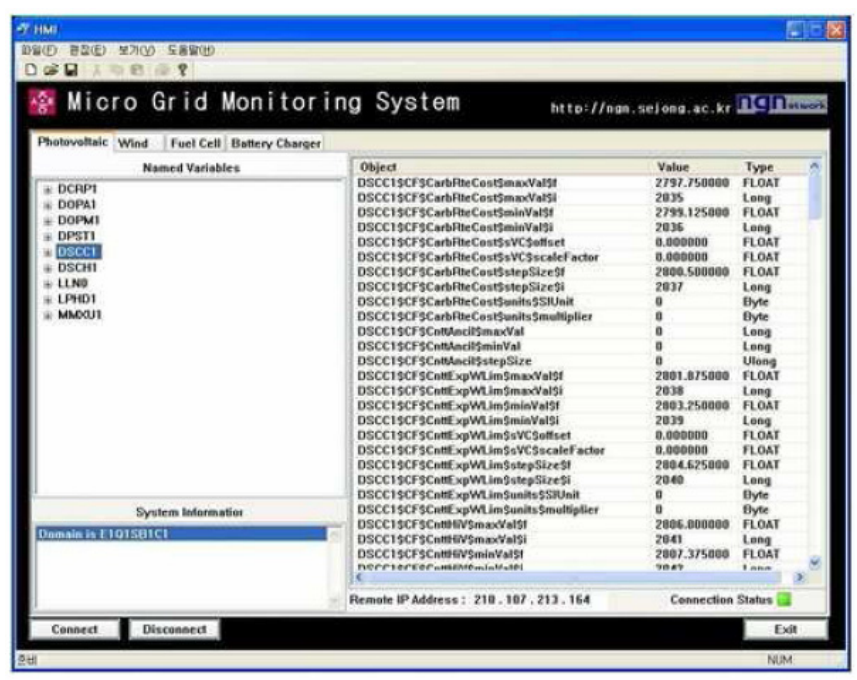

Fig. 4. Micro grid monitoring system

The Human Machine Interface (HMI) client system uses the IEC 61850 Abstract Communication Service Interface (ACSI) services mapped to manufacturing message specification (MMS) services to communicate with DER units. The ACSI services used in the micro grid monitoring system are summarized in Table 1. The micro grid monitoring system can change the value of the permitted data model and send control commands to the inverter for possible operation to each DER unit. 
Table 1. Mapping between ACSI services and MMS services

\begin{tabular}{c|c}
\hline ACSI Service & MMS Service \\
\hline Associate & Initiate \\
\hline GetServerDirectory & GetNamedList \\
\hline SetLogicalDeviceDirectory & GetNamedList \\
\hline GetDatasetDirectory & GetNamedList \\
\hline GetDataDefinition & GetVariableAccessAttribute \\
\hline GetDataDirectory & GetVariableAccessAttribute \\
\hline GetDataValue & Read \\
\hline SetDataValue & Write \\
\hline Release & Conclude \\
\hline
\end{tabular}

\subsection{Gateway}

The software architecture of the deployed gateway is shown in Fig. 5. The gateway plays two roles: as the master node of the DER control unit over the serial link and as an IEC 61850 server for the micro grid monitoring system over the Ethernet link. Different IEC 61850 servers are implemented for different DER control units. The gateway has two communication interfaces, i.e., the serial interface and the Ethernet interface. The gateway receives data from a DER controller using the serial link with the proprietary protocol developed in the present work, after which it transfers data to the micro grid monitoring system using the Ethernet with the IEC 61850 protocol. As long as data mapping between the proprietary protocol and the IEC 61850 data model is possible, the proposed system architecture can be extended to any other protocol, e.g., DNP, ModBus, and so on.

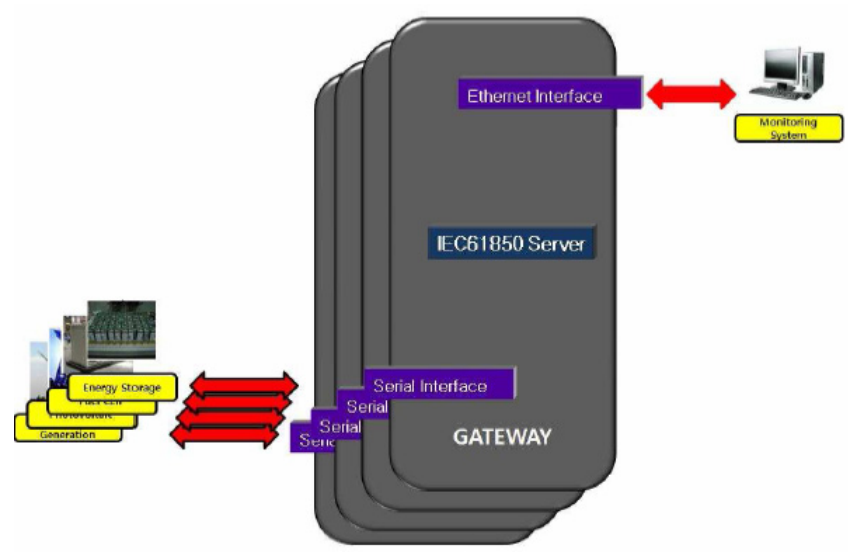

Fig. 5. Software architecture of a gateway

In the initial run of the gateway, it imports a configured IED description (CID) file based on substation configuration language (SCL) to form its own data objects. Of the DER units, the photovoltaic, fuel cell, and battery charger were modeled using the logical nodes defined in IEC 61850, while the wind unit was modeled using the logical nodes defined in IEC 61400-25-3 (Table 2). The mapping data based on the IEC 61850 data model for the wind DER unit is presented in Table 3 as an example.
Table 2. Data modeling of the DER nodes

\begin{tabular}{c|c|c}
\hline Nodes & LN & LN Description \\
\hline \multirow{4}{*}{ Fuel Cell } & DFCL & Fuel cell controller \\
\cline { 2 - 3 } & DSTK & Fuel cell stack \\
\cline { 2 - 3 } Photovoltaic & DFPM & Fuel processing module \\
\cline { 2 - 3 } & DPVM & Photovoltaic module rating \\
\cline { 2 - 3 } & DPVA & Photovoltaic array characteristics \\
\cline { 2 - 3 } & DPVC & Photovoltaic array controller \\
\hline \multirow{4}{*}{ Battery } & DTRC & Tracking controller name \\
\cline { 2 - 3 } & DBAT & Battery systems \\
\hline \multirow{5}{*}{ Wind } & WTUR & Wind turbine general information \\
\cline { 2 - 3 } & WTRM & Wind turbine transmission information \\
\cline { 2 - 3 } & WGEN & Wind turbine generator information \\
\cline { 2 - 3 } & WCNV & Wind turbine converter information \\
\cline { 2 - 3 } & WNAC & Wind turbine nacelle information \\
\cline { 2 - 3 } & WYAW & Wind turbine yawing information \\
\cline { 2 - 3 } & WTOW & Wind turbine tower information \\
\cline { 2 - 3 } & WMET & Wind turbine plant meteorological \\
& information \\
\cline { 2 - 3 } & WALM & Wind turbine plant alarm information \\
\cline { 2 - 3 } & WAPC & Wind turbine active power control \\
\cline { 2 - 3 } & WRPC & Wind turbine plant reactive power \\
control
\end{tabular}

Table 3. Mapping of wind turbine data

\begin{tabular}{|c|c|c|}
\hline $\begin{array}{l}\text { DER } \\
\text { node }\end{array}$ & Data & IEC 61850 Data \\
\hline \multirow{7}{*}{ Wind } & $\begin{array}{c}\text { Wind } \\
\text { Speed }\end{array}$ & WNAC1\$MX\$WdSpd\$mag\$f \\
\hline & $\begin{array}{l}\text { A phase } \\
\text { Voltage }\end{array}$ & WGEN1\$MX\$StaPhv\$phsA\$cVal\$mag\$f \\
\hline & $\begin{array}{l}\text { B phase } \\
\text { Voltage }\end{array}$ & WGEN1\$MX\$StaPhv\$phsB\$cVal\$mag\$f \\
\hline & $\begin{array}{l}\text { C phase } \\
\text { Voltage }\end{array}$ & WGEN1\$MX\$StaPhv\$phsC\$cVal\$mag\$f \\
\hline & $\begin{array}{l}\text { A phase } \\
\text { Current }\end{array}$ & WGEN1\$MX\$StaA\$phsA\$cVal\$mag\$f \\
\hline & $\begin{array}{l}\text { B phase } \\
\text { Current }\end{array}$ & WGEN1\$MX\$StaA\$phsB \$cVal\$mag\$f \\
\hline & $\begin{array}{l}\text { C phase } \\
\text { Current }\end{array}$ & WGEN1\$MX\$StaA\$phsC\$cVal\$mag\$f \\
\hline
\end{tabular}

However, not all data used in the micro grid system are defined in IEC 61850. Some data used in the current micro grid system are missing. The present study used the GGIO logical node, which is meant for generic process $\mathrm{I} / \mathrm{O}$, for mapping of undefined data.

As for the hardware platform, the ColdFire MCF5484 Lite evaluation board by Freescale was used. To implement the IEC 61850 server, Linux BSP was used because IEC 61850 required a TCP/IP protocol stack as well as an ISO/OSI 8802-3 Ethertype. Linux BSP was installed with a v2.4.26 kernel. Basic hardware specifications for MCF 5484 Lite evaluation board included a 64 MB DDRSDRAM and $4 \mathrm{MB}$ of boot flash. Installation of the gateway and DER controller unit is shown in Fig. 6. The presented setup was duplicated for each DER unit, i.e., for photovoltaic, fuel cell, battery charger, and wind turbine.

The gateway sends report messages to the micro grid monitoring system when data received from a DER controller is different from what the gateway has or within the pre-defined period. Due to hardware limitation, the 
gateway can send report messages every $10 \mathrm{msec}$ on a minimum, translating to 100 report messages per second.

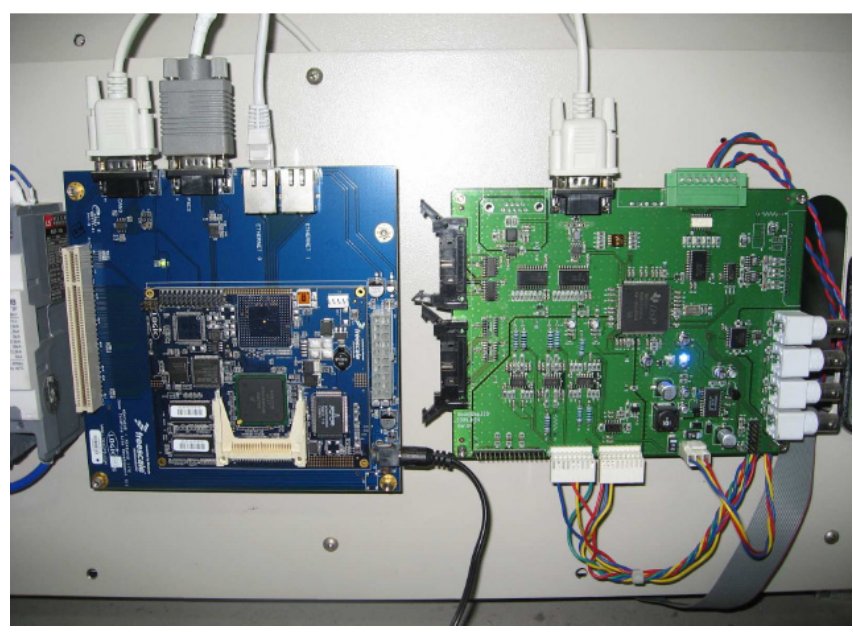

Fig. 6. Installed gateway with DER controller

\subsection{DER Controller}

The DER controller is connected to a DER node using hard-wire and implemented using a TMS320F28335 evaluation board from Texas Instrument. This board includes 16 channels of analogue to digital conversion (ADC), 12 ports of pulse width modulation (PWM), an encoder, and four channels of digital to analogue conversion (DAC) with a serial interface. The DER controller receives measured and status data from each DER unit and saves them in the internal memory space. Once the DER controller receives command (read) messages from the master node, i.e., the gateway, it transmits the corresponding data to that gateway.

\section{Conclusion}

To ensure the co-existence of different communication protocols in the current power industry as well as the successful operation of a smart grid, the issue of interoperability must be resolved. For years, the serial link has been one of the common communication links usedd in the power industry. IEC 61850, the communication protocol adapted for power utility automation system, however, uses Ethernet as a communication link with the data model. The Ethernet is a dominant communication protocol in the Internet with a very large bandwidth. However, new standards have been legislated recently, allowing up to $10 \mathrm{Gbps}$ of speed.

The present paper presented the deployment of IEC 61850-based micro grid system from the communication perspective. The proposed system consisted of a micro grid monitoring system, a protocol converter, and DER controllers with DER units. The DER controller collected data from DER units and sent them to the gateway using a serial link. The gateway converts data that have been mapped after the IEC 61850 data model, and sends them to the micro grid monitoring system. The deployed IEC 61850-based micro grid monitoring system successfully accomplished basic control and monitoring capability by virtue of a developed protocol converter. However, not all data used in the current micro grid system are mapped directly to the data models defined in IEC 61850. In this work, we used general I/O logical node to map undefined data as the standard allows it. Given the hardware limitation, the gateway can send a maximum of 100 IEC 61850 service messages per second to the monitoring system. Software optimization can solve a part of this problem.

The developed gateway platform has several communication interfaces aside from the serial link. It can be expanded to support other communication protocols, e.g., CAN or USB. In the future, the deployed micro grid system must be tested for stable operation.

\section{Acknowledgements}

The present work was financially supported by the Advanced Human Resource Development Program of the Ministry of Knowledge and Economy through the Research Center for Intelligent Microgrid in Myongji University and under the Convergence Information Technology Research Center support program (NIPA2011-C6150-1101-0003) supervised by the National IT Industry Promotion Agency.

\section{References}

[1] "Smart grid dictionary [online]," Available at http://www.smartgridlibrary.com/smart-grid-dictionary

[2] S. S. Venkata, A. Pahwa, R. E. Brown and R. D. Christie, "What future distribution engineers need to learn,", IEEE Trans. on Power Systems, vol. 19, no. 1, pp. 17-23, Feb. 2004

[3] F. M. Cleveland, "IEC 61850-7-420 communications standard for distributed energy resource (DER)," in Proceedings of IEEE PES general meeting, pp. 1-4, 2008

[4] IEC 61850-7-420 Ed. 1.0, “Communication networks and system in power utility automation - Part 7-420: Basic communication structure - distributed energy resources logical nodes," March 2009, Available at http://www.iec.ch.

[5] T. Skiei, S. Johannessen, and C. Brunner, "Ethernet in substation automation," IEEE Control Systems Mag., pp. 43-51, Jun. 2002.

[6] H. S. Yang and et. al., "Gigabit ethernet based substation," Journal of Power Electronics, pp. 100- 
108, Jan. 2009.

[7] Z. Yongli, W. Dewen, W. Yan, and Z. Wenqing, "Study on interoperable exchange of IEC 61850 data model," in Proc. of ICIEA, pp. 2724-2728, 2009.

[8] A. A. Zaidi and F. Kupzog, "Microgrid automation A self-configuring approach," in Proc. of INMIC, pp. 565-570, 2008

[9] P. M. Kanabar, M. G. Kanabar, W. El-Khattam, T. S. Sidhu, and A. Shami, "Evaluation of communication technologies for IEC 61850 based distribution automation system with distributed energy," in Proc. of PES, pp. 1-8, 2009.

[10] L. K. Siow, P. L. So, H. B. Gooi, F. L. Luo, C. J. Gajanayake, and Q. N. Vo, "Wi-Fi based server in microgrid energy management system," in Proc. of TENCON, pp. 1-5, 2009.

[11] Z. Bingquan, R. Yanming, and J. Jianning, "Strategy for implementation of IEC 61850 in substation automation system during transitional," in Proc. Of Automation of Electric Power Systems, pp. 54-57, 2005.

[12] J. W. Ginn, J. O.-M. David, D. F. Menicucci, T. Byrd Jr., S. Gonzalez, and J. D. Blevins, "Laboratory Tests of IEC DER Object Models for Grid Applications," Tech. Rep., Sandia National Laboratories, Feb. 2007.

[13] C. Binding, D. Gantenbein, B. Jansen, O. Sundström, P. B. Andersen, F. Marra, and C. Træholt, "Electric Vehicle Fleet Integration in the Danish EDISON Project - A Virtual Power Plant on the Island of Bornholm," Tech. Rep., IBM Research - Zurich and Technical University of Denmark, Jan. 2010.

[14] J.-H. Jeon, S.-K. Kim, C.-H. Cho, J.-B. Ahn, and E.-S. Kim, "Development of Simulator Systems for Microgrids with Renewable Energy Sources," Journal of Electrical Engineering \& Technology, vol. 1, no. 4, pp. 409-413, 2006.

[15] IEC 61850, "Communication networks and system in substation automation," 2002-2005, Available at http://www.iec.ch.

[16] IEC 61400-25-3, "Wind turbines - Part 25-3: Communications for monitoring and control of wind power plants - Information exchange models," Dec. 2006, Available at http://www.iec.ch.

[17] S.-J. Rim, S.-W. Zeng, and S.-J. Lee, "Development of an Intelligent Station HMI in IEC 61850 Based Substation," Journal of Electrical Engineering \& Technology, vol. 4, no. 1, pp. 13-18, 2009.

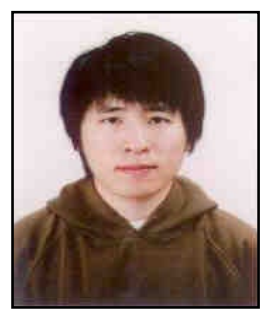

Byong-Kwan Yoo He received his B.S. degree in Computer Science and Engineering from Sejong University (Seoul, Korea) in 2010. He is currently working toward his M.S. degree at Sejong University. His research interests include power electronics application systems and communication systems based on IEC 61850 of the smart grid.

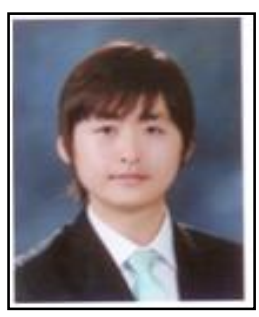

Seung-Ho Yang He received his B.S. in Computer Science and Engineering from Sejong University, Seoul, Korea in 2010. He is currently working toward his M.S. degree at Sejong University. His research interests include power electronics applications for Substation Automation System complying with IEC 61850, communication networks, and systems in substations.

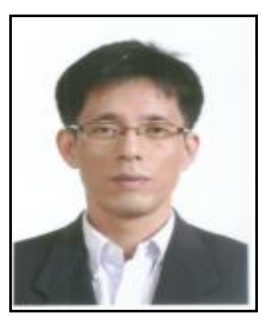

Hyo-Sik Yang $\mathrm{He}$ is an assistant professor at the Department of Computer Science and Engineering at Sejong University, (Seoul, Korea). Before he joined Sejong University, he was an assistant professor at Kyungnam University, Masan, Korea. He joined Sejong University in the fall semester of 2006. He served as a faculty research associate at Arizona State University until 2005. He received his B.E. degree in Information and Communication Engineering from Myongji University (Yongin, Korea) in 1998, and his M.S. and Ph.D. in Electrical Engineering from Arizona State University, (Tempe, AZ, U.S.A.), in 2000 and 2005, respectively. His research interests include wavelengthdivision-multiplexing all-optical networks, mobile ad-hoc networks, and smart grid.

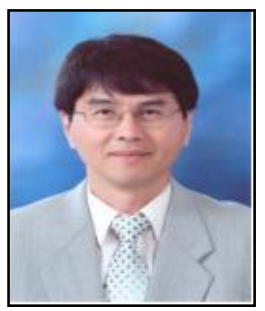

Byung-Moon Han $\mathrm{He}$ received his B.S. in Electrical Engineering from Seoul National University, Korea, in 1976, and his M.S. and Ph.D. from Arizona State University in 1988 and 1992, respectively. He worked at the Westinghouse Electric Corporation as a Senior Research Engineer in the Science and Technology Center. Currently, he is a Professor in the Department of Electrical Engineering, Myongji University, Korea. His research interests include power electronics applications for FACTS, custom power, and distributed generation.

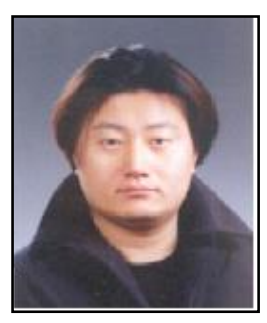

Yu-Seok Jeong He was born in Daegu, Korea, in 1971. He received his B.S., M.S., and Ph.D. degrees in Electrical Engineering from Seoul National University, (Seoul, Korea) in 1993, 1995 and 2005, respectively. He joined the Kia Motors Technical Center, Seoul, Korea, as a Research Engineer in 1995. In 2001 and 2002, he was a special student at the University of Wisconsin, Madison. During his doctoral 
course, he pursued fault-tolerant control and robust adaptive control of IPM synchronous machine drives in collaboration with GM. Following a one-year experience to develop a motor drive system for HEV/FCV applications at Hyundai Motor Company in 2005, he worked with the Department of Electrical Engineering of Myongji University (Gyeonggi-do, Korea) where he currently teaches. His research interests include modeling and digital control of power electronics and energy conversion.

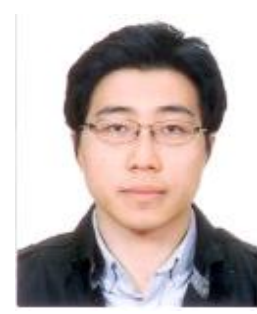

Won-Yong Kim He received his M.S. in Electrical Engineering from Myongji University, Korea, in 2011. $\mathrm{He}$ is currently a Ph.D. candidate at Myongji University. His research interests include power electronics applications for $\mathrm{HEV} / \mathrm{EV}$.

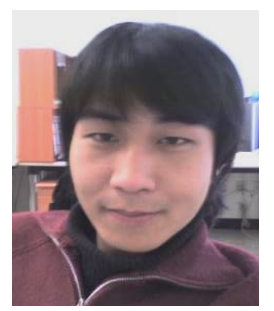

Kwang-Soo Jang He received his B.E. and the M.S. degrees in Computer Science and Engineering from Sejong University, Seoul, Korea, in 2008 and 2010, respectively. From 2010, he began working as a programming analyst for LG CNS. He joined the Smart BDP project in 2011 to help content providers deploy their services on LG media devices. His research interests are routing in mobile ad-hoc networks, smart grids, and the WEB OS. 